\title{
Photoacoustic trace gas detection of ethane using a continuously tunable, continuous-wave optical parametric oscillator based on periodically poled lithium niobate
}

\author{
M. M. J. W. van Herpen ${ }^{\text {a) }}$ \\ Molecular and Laser Physics, University of Nijmegen, 6525 ED Nijmegen, The Netherlands \\ Shaocheng $\mathrm{Li}$ \\ Applied Physics Department, Dalian University of Technology, Dalian, People's Republic of China \\ S. E. Bisson \\ Combustion Research Facility, Sandia National Laboratories, Livermore, California, 94550 \\ F. J. M. Harren \\ Molecular and Laser Physics, University of Nijmegen, 6525 ED Nijmegen, The Netherlands
}

(Received 17 April 2002; accepted for publication 24 June 2002)

\begin{abstract}
A $1.2 \mathrm{~W}$, continuous-wave, continuously-tunable, singly-resonant optical parametric oscillator (OPO) (idler tuning range 3.0-3.8 $\mu \mathrm{m}$ ), pumped by a $10 \mathrm{~W}$ continuous-wave Nd:YAG laser, is used in combination with a photoacoustic cell for the detection of ethane. An intracavity solid-state etalon (thickness $400 \mu \mathrm{m}$ ) was used to stabilize the OPO cavity and could be used to mode-hop tune the idler wavelength over $10 \mathrm{~cm}^{-1}$. The usefulness of the system was demonstrated by determining a detection limit for ethane down to 10 parts per trillion. The selectivity was achieved by making a 24 $\mathrm{GHz}$ wide pump laser scan over the ethane absorption line at $2996.9 \mathrm{~cm}^{-1}$, after which a Lorentzian fit determined the total area of the absorption signal. Both area value and peak value proved to be linearly depending on the ethane concentration. (C) 2002 American Institute of Physics.
\end{abstract}

[DOI: $10.1063 / 1.1500410]$

The advantage of infrared laser-based trace gas detection lies in its extreme sensitivity and specificity in tracing gaseous molecules with the possibility of rapid detection. ${ }^{1,2}$ For example, using laser-based photoacoustic spectroscopy, concentrations down to 10 part per trillion (ppt) for ethylene $\left(\mathrm{C}_{2} \mathrm{H}_{4}\right)$ and $100 \mathrm{ppt}$ for acetaldehyde ${ }^{3,4}$ can be measured. These features render infrared spectroscopic methods superior to gas chromatographs, mass spectroscopy, and classical infrared absorption techniques, which have orders of magnitude less sensitivity [5-10 parts per billion (ppb) for ethylene and $100 \mathrm{ppb}$ for acetaldehyde]. The performance of photoacoustic trace gas detection improves with higher laser power. That is why in the past high power gas lasers such as the $\mathrm{CO}_{2}$ laser $(9-11 \mu \mathrm{m}),{ }^{5,6}$ the $\mathrm{CO}$ laser $(5-8 \mu \mathrm{m}),{ }^{4,7}$ and the $\mathrm{CO}$ overtone laser $(2.8-4 \mu \mathrm{m})^{8}$ were used. These lasers allow the detection of a large number of environmental, biological, and medical relevant gases, ${ }^{1,5}$ but their disadvantage lies in their discrete line tunability. A continuously tunable laser source would allow more gases to be measured with increased sensitivity and would have a better selectivity between gases that have an absorption line close to each other. Good examples of tunable, mid-infrared sources are the quantum cascade distributed feedback lasers. ${ }^{9,10}$ For photoacoustic spectroscopy, optical parametric oscillators (OPOs) are a better choice, because they can give continuous tunability next to a high output power. ${ }^{11}$ Kühnemann et al. have demonstrated a detection limit of $0.5 \mathrm{ppb}$ for ethane with a low power $(40 \mathrm{~mW})$ singly resonant OPO (SRO) that is not

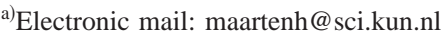

continuously tuned during the trace gas measurements. ${ }^{12}$ Here we demonstrate a detection limit of $0.01 \mathrm{ppb}$ with a high power OPO that is continuously tuned over $24 \mathrm{GHz}$ during the trace gas measurements.

The OPO is similar to what is described before. ${ }^{11,13}$ Using a periodically poled lithium niobate (PPLN) crystal with a fan out grating design (periods ranging from 29.3 to 30.1 $\mu \mathrm{m})$ a signal beam (wavelength $1.5-1.7 \mu \mathrm{m}$ ) and an idler beam $(3.0-3.8 \mu \mathrm{m})$ are generated via optical parametric conversion from a $1064 \mathrm{~nm}$ pump source. The pump source is a Nd:YAG Master Oscillator Power Amplifier system (M6000, Lightwave, CA) generating more than $10 \mathrm{~W} \mathrm{cw}$ at $1064 \mathrm{~nm}$. The pump-laser linewidth is $5 \mathrm{kHz}$ (over $1 \mathrm{~ms}$ ) with a frequency stability of $50 \mathrm{MHz} / \mathrm{h}$ and it has a transverse electromagnetic mode (TEM) $)_{00}$ spatial mode with a beam quality of $M^{2}<1.1$. The pump can be continuously tuned over 24 $\mathrm{GHz}$ with two reproducing mode-hops occurring backwards compared to the direction of tuning. ${ }^{11}$ For our SRO, a bowtie ring cavity is used to resonate the signal wavelength, while transmitting the idler and pump wavelengths. Single mode operation of this cavity is enhanced by means of an intracavity etalon.

An important consideration is whether to use an airspaced or solid state etalon. The advantage of using an airspaced etalon is that the losses are constant after inserting it, which reduces etalon mode hopping. ${ }^{14}$ The drawback of the air-spaced etalon is that (in our case) the OPO oscillation threshold increased considerably, resulting in a lower output power. Proper alignment of such an etalon is experimentally difficult and also very important to reduce losses. In addition, an air spaced etalon requires more intracavity space as com- 


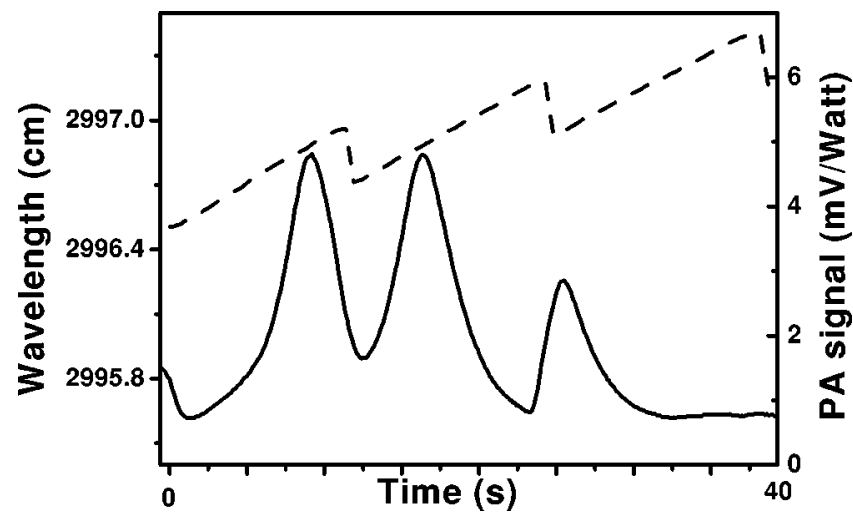

FIG. 1. A $24 \mathrm{GHz}$ pump scan of the OPO at the idler wavelength. The solid line shows the photoacoustic signal of a mixture of $3 \mathrm{ppm}$ ethane in nitrogen. The wavelength (dashed line) shows two mode hops which are caused by reproducible mode hops of the pump laser, which are always backwards compared to the direction of tuning.

pared to a solid etalon. For our actual spectroscopic trace gas experiments we do not tune the intracavity etalon, but instead we tune the Nd:YAG pump source, which will cause the idler frequency to tune as well. This means that the constant losses during etalon tuning are of minor importance to us, so we have decided to use a solid state etalon as this gives a higher output power.

According to previous attempts ${ }^{14}$ we used a $400-\mu \mathrm{m}-$ thick solid YAG etalon with a free spectral range of $207 \mathrm{GHz}$ (VLOC, New Port Richey, FL). The plane parallel ( $<10$ arc seconds), uncoated ( $R=8 \%$ ) YAG etalon appeared to be selective enough to stabilize the OPO cavity to a single mode, without increasing the threshold power much (from 3 to 4 $\mathrm{W})$. The idler output power went down from $1.5 \mathrm{~W}$ to $1.2 \mathrm{~W}$.

To mode-hop tune the OPO wavelength the YAG etalon can be angle rotated with a galvo driver. When the PPLN crystal is kept at a fixed periodicity during rotation of the etalon, a tuning range of $10 \mathrm{~cm}^{-1}$ can be covered for the idler wavelength. A significant change in idler output power is observed during rotation, which can be explained by walk off losses within the solid etalon. The mode-hop-tuning behavior of the OPO is similar as described by Bisson et al. ${ }^{14}$ When rotating the etalon the idler wavelength is tuning in steps of $0.02-0.1 \mathrm{~cm}^{-1}(0.6-3 \mathrm{GHz})$, depending on the number of cavity modes jumped. Sometimes, several mode hops are observed giving steps of more than $1 \mathrm{~cm}^{-1}$. In future experiments we plan to use higher finesse YAG etalons (reflectivity $R>20 \%$ ) to decrease the mode-hop step size.

For photoacoustic detection of ethane $\left(\mathrm{C}_{2} \mathrm{H}_{6}\right)$, we coarse tuned the OPO to the center of the absorption line at $2996.9 \mathrm{~cm}^{-1}$ by adjusting the fan-out PPLN crystal and by tuning the intracavity solid-state etalon. The pump laser was then continuously tuned over $24 \mathrm{GHz}$ to record the entire absorption peak (Fig. 1). A wavemeter (Burleigh WA-1000 IR) was used to record the idler wavelength with a resolution of $0.01 \mathrm{~cm}^{-1}$.

The OPO idler beam was directed through a photoacoustic cell in which a mixture of ethane in nitrogen was kept at STP. The photoacoustic cell is similar to a design described earlier, ${ }^{3}$ but we increased the length of the acoustic resonator from 100 to $300 \mathrm{~mm}$ and reduced the radius from 3 to $2 \mathrm{~mm}$, which improved the sensitivity by a factor of $2.6 .^{3}$ The

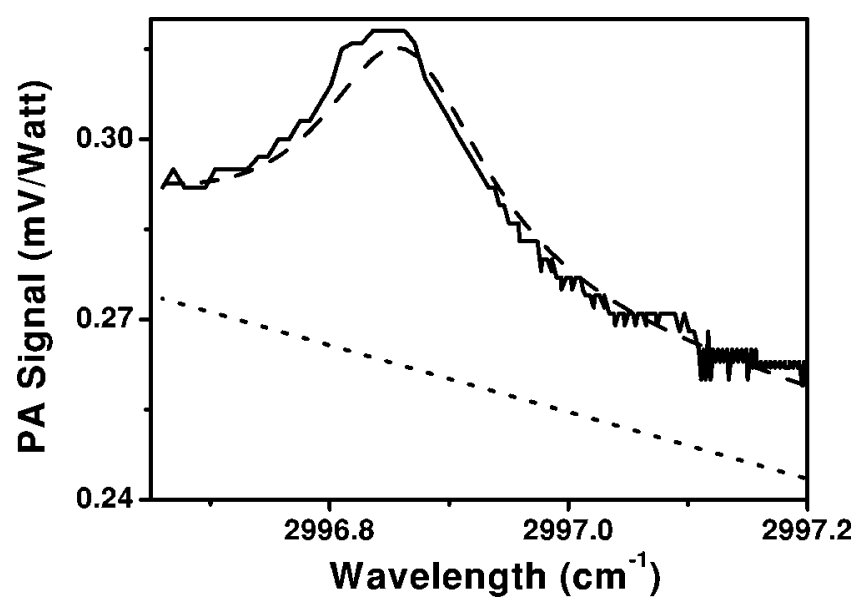

FIG. 2. Photoacoustic signal of the ethane absorption line at $2996.9 \mathrm{~cm}^{-1}$ for a mixture of $0.4 \mathrm{ppb}$ ethane in nitrogen. A Lorentzian fit (dashed line) with a linearly decreasing background (dotted line) has been plotted through the experimental data (solid line). Evident are the microphone noise ( $5 \mu \mathrm{V} / \mathrm{Hz}^{1 / 2}$ ) and the high background signal $(0.3 \mathrm{mV} / \mathrm{W})$. Due to this wavelength-dependent background signal it is hard to use solely the peak value of the absorption at this ethane concentration, but the Lorentzian fit is able to find the background signal very well. With background subtracted the photoacoustic signal is $0.04 \mathrm{mV} / \mathrm{W}$.

acoustic resonator is excited in the first longitudinal mode at a resonance frequency of $560 \mathrm{~Hz}$. In addition, we used three Knowless IK3024 electret microphones instead of one.

Figure 1 shows an example of an OPO scan acquired by tuning of the pump source. Although two mode-hops were observed in this scan, they were reproducible from scan to scan and could be removed from the data by plotting the recorded wavelength against the measured photoacoustic signal. These mode hops always occurred opposite to the direction of tuning and were attributed to mode hops in the pump source. An example of a scan of $0.4 \mathrm{ppb}$ ethane in nitrogen is shown in Fig. 2, which shows the pressure broadened Lorentzian least-squares fit of the ethane absorption peak. For this fit, a linearly decreasing background signal was added to the Lorentzian profile. The picture shows that this fit can be used very well to find the background signal and to fit the actual absorption signal. With the fitted background subtracted, the total area beneath the Lorentzian curve can be used as a value for the strength of the absorption and thus the concentration.

To find the ethane detection limit for our system we have measured the absorption of ethane at $2996.9 \mathrm{~cm}^{-1}$ for different concentrations of ethane in nitrogen. Using two mass flow controllers, pure nitrogen gas was mixed with 20 ppm and $0.8 \mathrm{ppb}$ ethane/nitrogen mixtures to obtain ethane concentrations ranging from $20 \mathrm{ppm}$ to $0.01 \mathrm{ppb}$. Figure 3 shows two calibration lines: one for the peak values of the absorption line and one for the total area under the Lorentzian fit. Both values show a linear behavior with the concentration, which stops being linear at the noise level and becomes a horizontal line. The detection limit (signal-to-noise ratio $\mathrm{SNR}=1$ ) using the peak values was found to be $0.1 \mathrm{ppb}$, and was limited by the microphone noise of $5 \mu \mathrm{V} / \mathrm{Hz}^{1 / 2}$. Using the peak height was made difficult due to a varying background signal around $0.3 \mathrm{mV} / \mathrm{W}$ (Fig. 2). In this case the fitted background signal could be subtracted, but without continuous tuning this would not have been possible and 


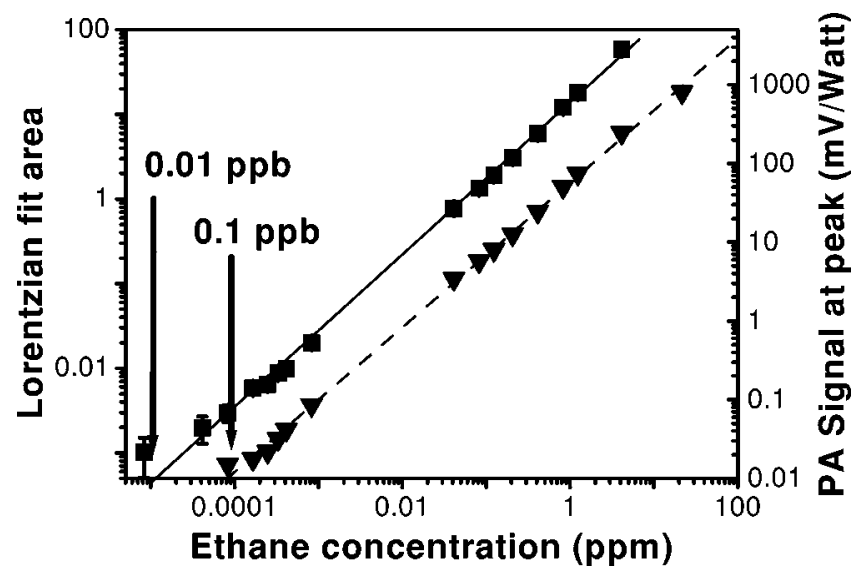

FIG. 3. The detection limit of ethane is determined by measuring the peak height (triangles + dotted line) and the area of the fit of the absorption (squares + solid line) at $2996.9 \mathrm{~cm}^{-1}$ for different concentrations of ethane in nitrogen. The detection limit is determined by the noise of the system, which is used as the horizontal axis. Using the peak values a detection limit of $0.1 \mathrm{ppb}$ was found. With the Lorentzian fit area a detection limit of 0.01 $\mathrm{ppb}$ is found.

would have resulted in a lower detection limit of $5 \mathrm{ppb}$. The detection limit using the Lorentzian fit was found to be 0.01 $\mathrm{ppb}(\mathrm{SNR}=1)$, which was limited by the noise of the total area under the Lorentzian curve (scan time $40 \mathrm{~s}$ ). The noise in the area value was determined by measuring the area values for pure nitrogen. Because the Lorentzian fit helps to find the background signal, a much better detection limit was obtained using the Lorentzian fit area values. Using the Hitran database ${ }^{15}$ the maximum absorption strength of ethane at $2996.9 \mathrm{~cm}^{-1}$ was determined to be $33.4 \mathrm{~atm}^{-1} \mathrm{~cm}^{-1}$. Combined with the detection limit of $0.01 \mathrm{ppb}$, this results in a detection limit of $3 \times 10^{-10} \mathrm{~cm}^{-1}$ (scan time $40 \mathrm{~s}$ ).

While we have demonstrated the use of a PPLN OPO for trace gas detection of ethane, the broad tunability allows a wide range of different gases to be detected in the mid-IR (3.0-3.8 $\mu \mathrm{m}$, e.g., vibrational $\mathrm{C}-\mathrm{H}$-stretch of molecules). Due mainly to the higher power, this system has a much better sensitivity compared to other OPO systems. ${ }^{12}$ In addition this system has a better specificity because it is continuously tunable. We demonstrated that the detection limit could still be reached if the background signal is varying. In future we expect to apply the system to various applications in biology and medicine. ${ }^{1,6,12}$

${ }^{1}$ F. J. M. Harren, G. Cotti, J. Oomens, S. te Lintel Hekkert, Photoacoustic Spectroscopy in Trace Gas Monitoring, in Encyclopedia of Analytical Chemistry, edited by R. A. Meyers (Wiley, Chichester, 2000), pp. 22032226.

${ }^{2}$ G. Berden, R. Peters, and G. Meijer, Int. Rev. Phys. Chem. 19, 565 (2000).

${ }^{3}$ F. G. C. Bijnen, J. Reuss, and F. J. M. Harren, Rev. Sci. Instrum. 67, 2914 (1996).

${ }^{4}$ H. Zuckermann, F. J. M. Harren, J. Reuss, and D. H. Parker, Plant Physiol. 113, 925 (1997)

${ }^{5}$ M. W. Sigrist, in Air Monitoring by Spectroscopic Techniques, edited by M. W. Sigrist (Wiley, New York, 1994), Vol. 27, pp. 163-238.

${ }^{6}$ F. J. M. Harren, R. Berkelmans, K. Kuiper, S. te Lintel Hekkert, P. Scheepers, P. Hollander, R. Dekhuijzen, and D. H. Parker, Appl. Phys. Lett. 74, 1761 (1999).

${ }^{7}$ S. T. Persijn, R. H. Veltman, J. Oomens, F. J. M. Harren, and D. H Parker, Appl. Spectrosc. 54, 62 (2000).

${ }^{8}$ A. A. E. Martis, S. Büscher, F. Kuhnemann, and W. Urban, Instrum. Sci. Technol. 26, 177 (1998).

${ }^{9}$ C. Gmachl, D. L. Sivco, R. Colombelli, F. Capasso, and A. Y. Cho, Nature (London) 415, 883 (2002).

${ }^{10}$ M. Beck, D. Hofstetter, T. Aellen, J. Faist, U. Oesterle, M. Ilegems, E. Gini, and H. Melchior, Science 295, 301 (2002).

${ }^{11}$ M. M. J. W. van Herpen, S. te Lintel Hekkert, S. E. Bisson, and F. J. M. Harren, Opt. Lett. 27, 640 (2002).

${ }^{12}$ F. Kühnemann, K. Scheider, A. Hecker, A. A. E. Martis, W. Urban, S. Schiller, and J. Mlynek, Appl. Phys. B: Lasers Opt. 66, 741 (1998).

${ }^{13}$ P. E. Powers, T. J. Kulp, and S. E. Bisson, Opt. Lett. 23, 159 (1998).

${ }^{14}$ S. E. Bisson, K. M. Armstrong, T. J. Kulp, and M. Hartings, Appl. Opt. 40, 6049 (2001).

${ }^{15}$ The Hitran database, URL: http://cfa-www.harvard.edu/HITRAN/ 